\title{
Versions of the tools
}

- bedtools 2.27 .1

- bowtie: 1.2 .2

- bwa: 0.7.17-r1188

- FASTX Toolkit: 0.0.14

- Python: 2.7.16

- R: 3.6.1

- samtools: 1.9

- ShortStack: 3.8.5

\section{Releases of the genome assemblies}

- Arabidopsis thaliana: TAIR10

- Danio rerio: GRCz10

- Homo sapiens: GRCh38

- Sus scrofa: 11.1

\section{Scripts}

\section{Trimming and cleaning}

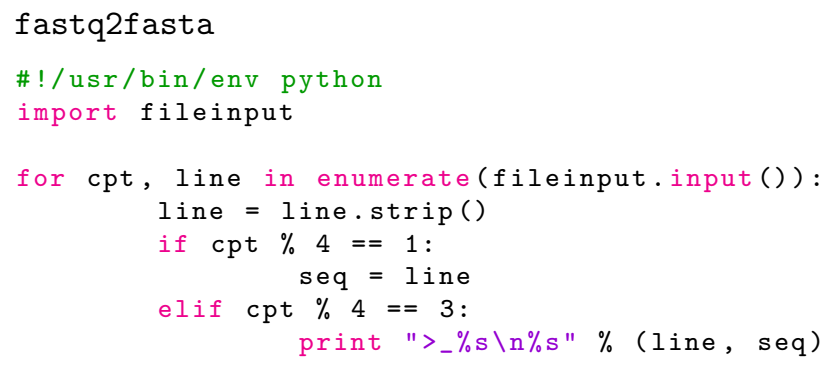




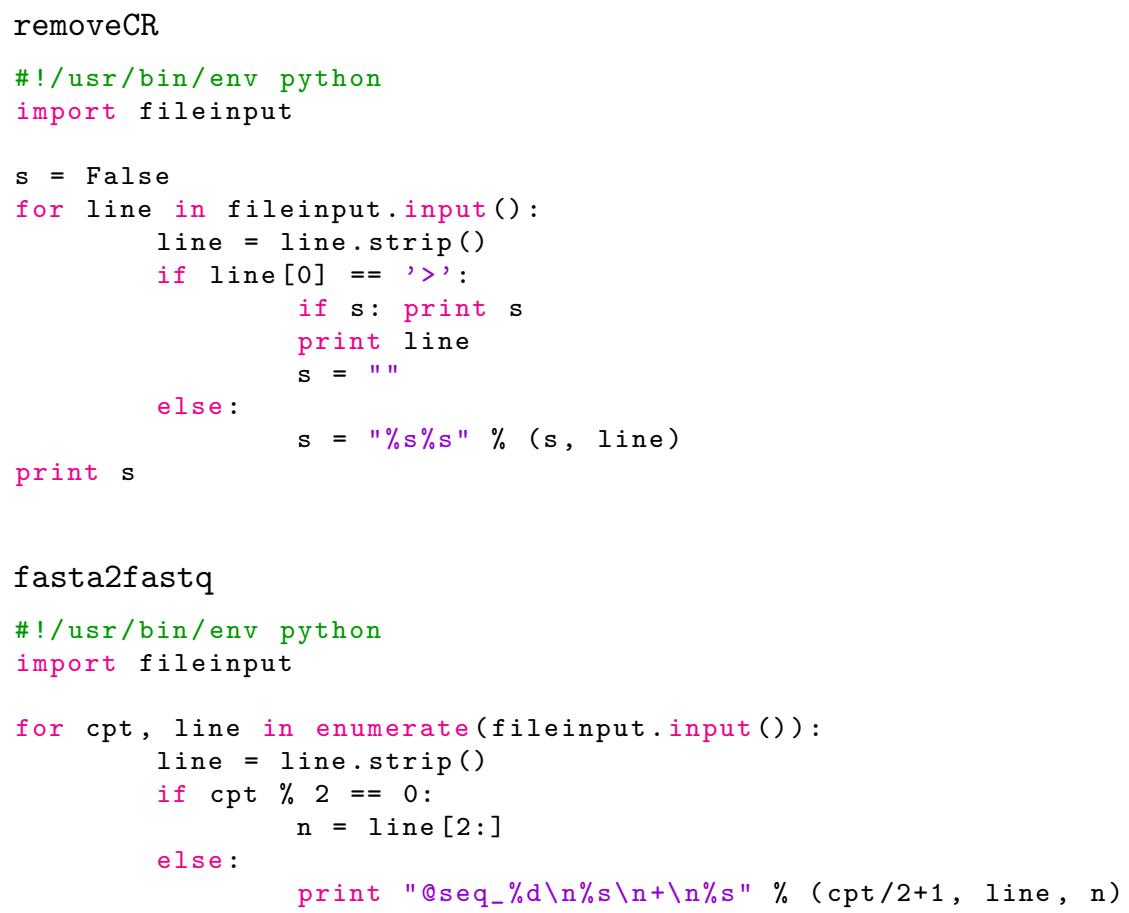

\section{Pipe-line}

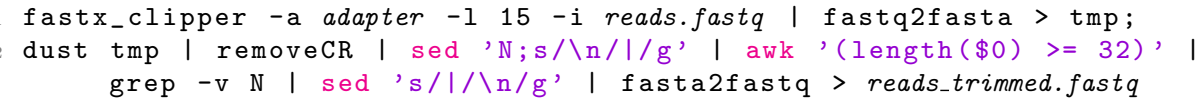

\section{Mapping}

\section{Mapping with BWA}

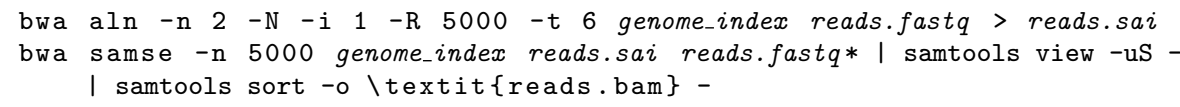

Mapping with bowtie

1 bowtie -S - a --best --strata -m 20 - p 6 genome_index reads.fastq । addNH I samtools view -uS - I samtools sort -o reads.bam -

\section{Quantification strategies}

\section{mmannot}

1 /usr/bin/time -o results.time mmannot -c configuration_file - a annotation.gtf $-\mathrm{r}$ reads.bam $-10.6-\mathrm{p}-\mathrm{y}$ strategy $-\mathrm{m}$ results_stats1.csv $-\mathrm{M}$ results_stats2.csv > results.csv 
The strategy can be unique, ratio, random, or default.

MMR MMR only works with the BAM files produced by bowtie.

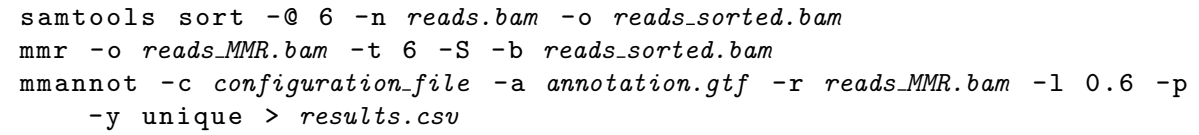

\section{ShortStack}

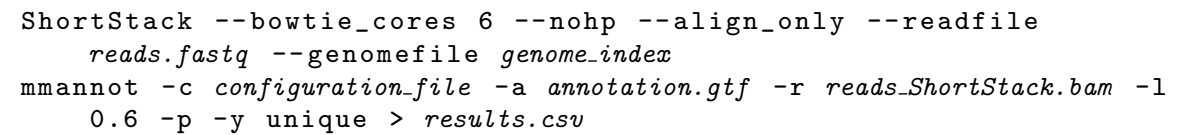

Extracting annotation of sRNAs from standard GFF/GTF files This was used for the Arabidopsis thaliana dataset. It has been adapted to other datasets.

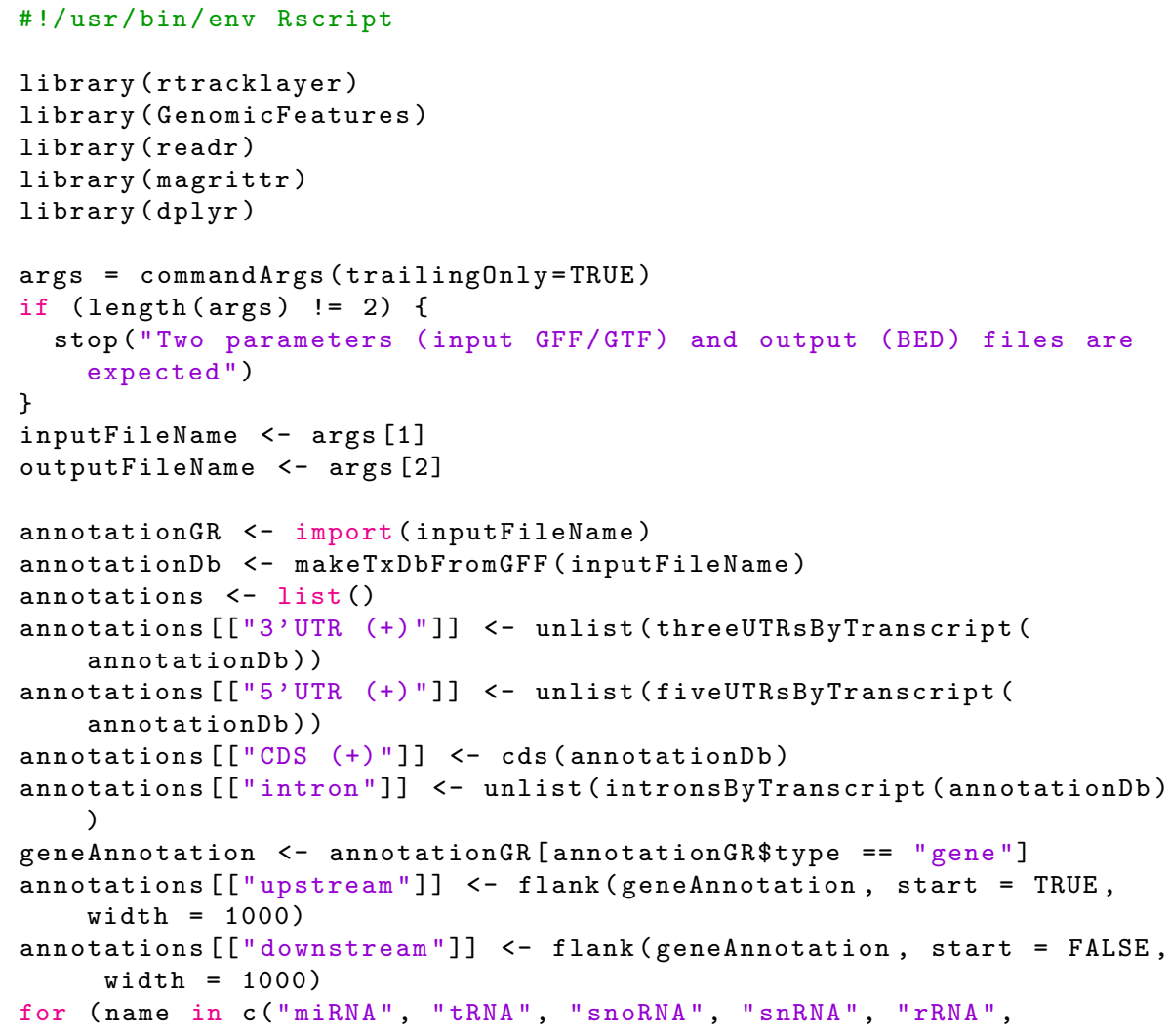




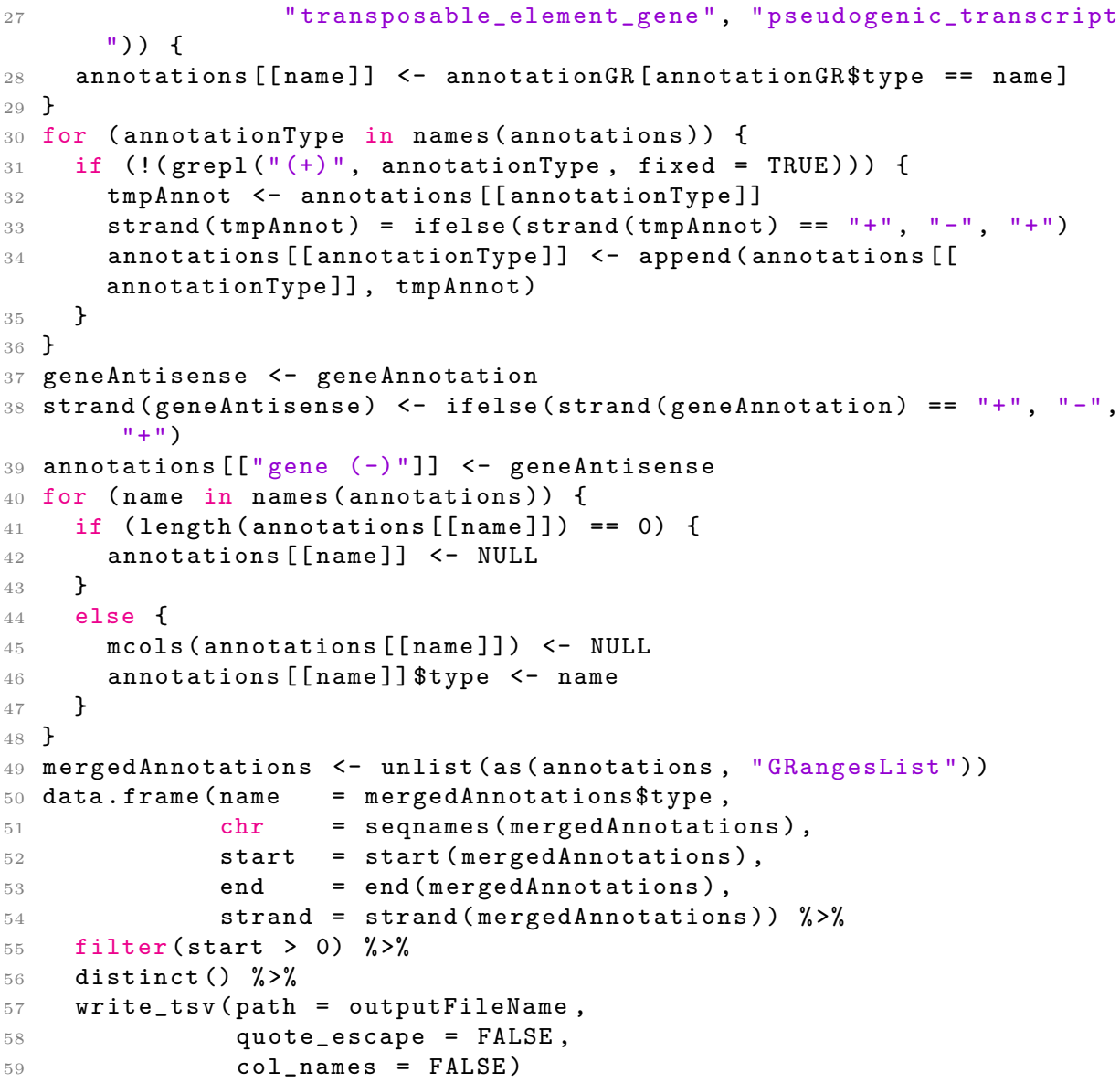

\section{FeatureCounts}

featureCounts -a annotation.saf -F SAF reads.bam --frac0verlap 0.6 -o output.fc $-\mathrm{L}-\mathrm{s} 1$

Generating a database of sequences of sRNAs and the genome

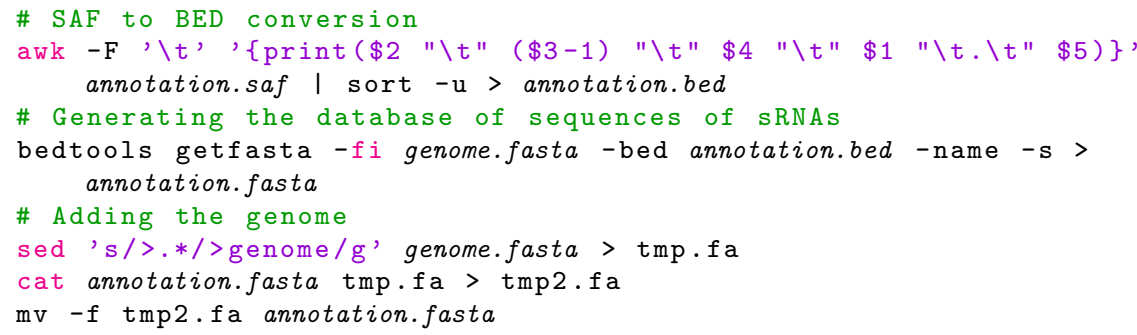

